\title{
Erratum
}

Med Klin Intensivmed Notfmed 2019 • 114:525 https://doi.org/10.1007/s00063-019-00606-w Online publiziert: 20. August 2019

(c) Springer Medizin Verlag $\mathrm{GmbH}$, ein Teil von Springer Nature 2019

\section{Erratum zu:}

Med Klin Intensivmed Notfmed 2019 https://doi.org/10.1007/s00063-0180516-9

In - Tab. 3 war die Dosierungsempfehlung für L-Thyroxin zur Indikation therapierefraktäre Herzinsuffizienz nicht korrekt. Bitte beachten Sie diese korrigierte Fassung.

\section{S.-O. Kuhn • K. Hahnenkamp}

Klinik für Anästhesiologie, Anästhesie, Intensiv-, Notfall- und Schmerzmedizin, Universitätsmedizin Greifswald, Greifswald, Deutschland

\section{Erratum zu:}

\section{Intensivmedizinisches Management des potentiellen Organspenders}

\section{Korrespondenzadresse}

\section{Dr. S.-0. Kuhn}

Klinik für Anästhesiologie, Anästhesie, Intensiv-, Notfall- und Schmerzmedizin, Universitätsmedizin Greifswald

Ferdinand-Sauerbruch-Straße, 17475 Greifswald, Deutschland

sven-olaf.kuhn@uni-greifswald.de
Tab. 3 Medikamentöse Therapieempfehlung (Auswahl)

\section{Indikation}

Hypotonie

infolge erniedrigter systemisch

vaskulärer Resistenz

\section{Hypotonie}

infolge Links- und Rechtsherzinsuffizi-

enz mit erhöhtem linksventrikulärem

Füllungsdruck und Erniedrigung des

Herzzeitvolumens

Diabetes insipidus centralis

Reduktion des Katecholaminbedarfs

Therapie der Hyperinflammation

Therapierefraktäre Herzinsuffizienz

Hyperglykämie
Desmopressin (DDAVP)

Substanz

Noradrenalin

Vasopressin

(Arginin-Vasopressin)

Dobutamin

0,5-5I.E./h

$2-10 \mu \mathrm{g} / \mathrm{kg}$ und Minute

Dosierungsempfehlung

$0,01-0,2 \mu \mathrm{g} / \mathrm{kg}$ und Minute ggf. Repetitionsdosen

Methylprednisolon $250 \mathrm{mg}$ Bolus,

$100 \mathrm{mg} / \mathrm{h}$

L-Thyroxin

Insulin
$20 \mu$ i.v. als Bolus gefolgt von $10 \mu \mathrm{g} / \mathrm{h}$ i.v.

1-10I.E./h

\section{Bemerkung}

Cave! Zunahme der pulmonalen Permeabilität und der koronaren/mesenterialen Vasokonstriktion

Reiner Vasokonstriktor, Kombination mit Noradrenalin, Reduktion der Noradrenalindosis

Mittel der ersten Wahl beim kardiogenen Schock zur Frequenzsteigerung (Atropinresistenz beim Hirnfunktionsverlust)

Synthetisches Analogon des antidiuretischen Hormons (ADH)

Nach Feststellung des irreversiblen Hirnfunktionsverlusts

Therapieversuch

Ziel: $4-10 \mathrm{mmol} / \mathrm{l}$

Die Online-Version des Originalartikels ist unter https://doi.org/10.1007/s00063-018-0516-9zu finden. 